\title{
Fruit juice consumption is associated with intakes of whole fruit and vegetables, as well as non-milk extrinsic sugars: a secondary analysis of the National Diet and Nutrition Survey
}

\author{
S. Gibson ${ }^{1}$ and C.H.S. Ruxton ${ }^{2}$ \\ ${ }^{1}$ SiG-Nurture Ltd, 11 Woodway, Guildford, Surrey, GU1 2TF, UK and ${ }^{2}$ Nutrition Communications, 26 East Road, \\ Cupar, KY15 4HQ, UK
}

Pure fruit juice (PFJ) contains naturally occurring 'free' sugars, providing $8-14 \%$ of daily intakes depending on the age group ${ }^{(2)}$. Free sugars are now the target of a challenging average population intake of $\leqslant 5 \%$ energy ${ }^{(1)}$, i.e. around $30 \mathrm{~g}$ daily for an adult. While concerns have been raised about the sugar content of PFJ, $150 \mathrm{ml}$ counts as one portion of fruit and PFJ continues to be acknowledged as a valid option within 5-A-Day messaging ${ }^{(3)}$.

A secondary analysis was conducted on 2967 participants aged 11 to 99 years from the National Diet and Nutrition Survey (20082012) as this is the age range to which the 5-A-day message applies. Dietary data were collected using a 4-day food record with estimated portion sizes. The aim of the analysis was to examine associations between PFJ consumption and 5-A-Day compliance, whole fruit and vegetable (F\&V) consumption, and non-milk extrinsic sugar (NMES) intakes (similar to free sugars).

Mean PFJ consumption was $83 \mathrm{ml} / \mathrm{d}$ in participants aged $11-18$ y (53\% were consumers) and $52 \mathrm{ml} / \mathrm{d}$ in those aged 19-64 y (39\% were consumers). Orange and apple were the most commonly consumed PFJs. After grouping participants by PFJ intake, associations were investigated with whole F\&V consumption (excluding composite dishes), as well as with NMES intakes as a proportion of daily total energy (see table below).

\begin{tabular}{|c|c|c|c|c|c|c|}
\hline \multirow[b]{2}{*}{ PFJ intake (mlld) \% of population } & \multicolumn{2}{|c|}{ Adults (19-64y) } & \multicolumn{4}{|c|}{ Young people (11-18y) } \\
\hline & 0 & $\leqslant 150 \mathrm{ml}$ & $>150 \mathrm{ml}$ & 0 & $\leqslant 150 \mathrm{ml}$ & $>150 \mathrm{ml}$ \\
\hline & 61 & 28 & 11 & 47 & 34 & 19 \\
\hline Whole vegetable intake $(\mathrm{g} / \mathrm{d})$ & 171 & 199 & 204 & 108 & 113 & 122 \\
\hline Whole fruit intake $(\mathrm{g} / \mathrm{d})$ & 94 & 126 & 130 & 50 & 68 & 81 \\
\hline$\%$ energy from NMES & $10 \cdot 6$ & $11 \cdot 9$ & $15 \cdot 2$ & $14 \cdot 3$ & $15 \cdot 5$ & $18 \cdot 1$ \\
\hline$n$ & 1305 & 550 & 228 & 449 & 259 & 176 \\
\hline
\end{tabular}

All linear associations were statistically significant (ANOVA) suggesting that higher PFJ consumption was associated with higher intakes of F\&V, but also higher NMES intakes. The small differences in NMES intake between non-consumers and those who consumed $\leqslant 150 \mathrm{ml}$ PFJ were only statistically significant in adults. On a positive note, PFJ consumers were significantly more likely to reach 5-A-DAY (39\% vs. $23 \%$ of non-consumers). Previous work has shown that PFJ consumers tend to have a lower BMI than non-consumers ${ }^{(4)}$.

In conclusion, PFJ intake appears to be a marker of a 'health-conscious' diet as well as a distinct contributor to 5-A-day (mean from PFJ $=0.4$ portion in adults and 0.6 portions in young people). Consuming up to $150 \mathrm{ml} / \mathrm{d}$ PFJ marginally increased NMES in adults but not in young people, suggesting this level of intake offers a benefit in terms of 5-A-day compliance, particularly since only $9 \%$ of children and $32 \%$ of adults achieve the F\&V target.

This work was funded by the British Fruit Juice Association.

1. Scientific Advisory Committee on Nutrition (2015) Carbohydrates and health. London: SACN.

2. Bates B et al. (2014). National Diet and Nutrition Survey years 1-4. London: FSA.

3. Public Health England (2015) Sugar reduction. The evidence for action. London: PHE.

4. Gibson S et al. (2015) Ann Nutr Metab 67 (supp 1): abstract 149/1262. 